\title{
Gender and Hedging Behavior: An Analysis of Functions of Hedges Employed by Women in Chinese Conversations
}

\author{
Yijia Chai ${ }^{1, *}, \dagger$ \\ ${ }^{I}$ Faculty of Humanities and Social Science, Newcastle University, NE1 7RU, Newcastle, UK \\ *Corresponding author. Email: charlottechai@m.poe.edu.pl
}

\begin{abstract}
Hedges have been regarded as signals only for tentativeness in expressions and stereotypically seen as a feature of women's speech since Lakoff publicized her work on features of women's language. This argument has been debated by Jennifer Coates by exploring the multi-functions of hedges in the usage of female speakers and elucidating the reasons why women tend to use them. There are four functions of hedges proposed by Coates: the expression of doubt and confidence, sensitivity to others' feelings, researching for the right word and avoidance of expert status. This research is carried based upon the categorization of Coates. In this study, the author investigated, in the context of Chinese podcast shows, the differences of function-related features of hedges in women's talk between mixed-sex conversations and same-sex conversations. The results showed that (1) overall, the subject showed more hedging behaviors when men were present; (2) all the four functions proposed by Coates displayed in the database; (3) the subject used more hedges in mixed-sex conversations than in same-sex conversations for expressing doubt and confidence, showing sensitivity to others' feelings and searching for the right word, but more hedges in same-sex conversations than in mixed-sex ones for showing the avoidance of playing the expert. In addition to this, an elaborative analysis of speech examples was given to show how hedges function as a facilitative role in women's interactions with different sexes in Chinese. The analysis showed that women were able to achieve interpersonal closeness and create a collaborative floor for everyone to participate in conversations.
\end{abstract}

Keywords: Hedge, Function of hedge, Sex differences in language, Women's speech, Addressee's gender

\section{INTRODUCTION}

Hedges are linguistic devices used to attenuate the full semantic value of a proposition or to damp down the force of a speech act [1]. Hedges and hedging behaviors have been seen as a crucial aspect for research on sex differences in language use since 1975, when Lakoff's masterpiece Language and Women's Place was published [2]. In her work, Lakoff maintains that women use more hedges than men because women are uncertain of their utterance. Research on sex differences in language use is not only an interest out of social and anthropological linguists curiosity, but also a way to understand discrepancies in respect of women and men's social roles, their power status and even different world values, as all of those factors can be communicated through language $[3,4]$.
A large number of studies show agreement with Lakoff's findings and conclusion [5-7]. However, through her exploration of hedges' interactional functions, Coates states that hedges per se are multi-functional in conversations, and this "belie(s) the idea that women are unassertive because they use more hedges than men" [8,9]. Similarly, Holmes emphasized the importance of understanding hedges' functional rationale when researchers examine similarities and differences in women's and men's speech [11]. The present study reports statistical results that show marked differences in function-related features of hedges in women's talk in mixed-sex conversations and same-sex conversations and offers some alternative interpretations in contrast to traditional ones that elucidate women's use of hedges as tentativeness and feminine weakness. This study also displays elaborations of excerpts from the database to show how women successfully use hedges as multi-functional rhetorical strategies to achieve their interactional goals. 
Previous literature on language, communication and gender reveals that there has been considerable research on features of hedges and hedging behaviors employed in women's and men's speech. Gender-related features in terms of whether women use more hedges (or specific linguistic devices used as hedges) than men have been extensively studied. Some studies, such as McMillan and his colleagues' study in a problem-solving context, shows women do use more linguistic forms to suggest their uncertainty [3]. Similarly, in Japanese conversations, Lauwereyns shows that hedges are more often used by female speakers [5]. By contrast, Precht finds that there is no significant difference between men and women's use of stance, of which hedges are an essential part, and in Dubar's study, the results are even contradictory, which is that women use fewer you know to hedge than men do on a British radio station [11, 12].

Other aspects which have been emphasized to be taken into consideration are the functional diversity of linguistic forms across different social and cultural contexts and contextual variables such as addresses' gender. Coates and Holmes have made a prominent contribution to demonstrating multiple functions of hedges $[8,10]$. Notably, Coates focuses on the multi-functional role of hedges in women's talk and offers reasons to explain why women use them. She has revealed there are four main functions of hedges: to express uncertainty and confidence, to show sensitivity to others' feelings, to search for the right words and to avoid taking on the role of expert. She interprets those hedging behaviors of women as women's attempts to show their politeness when discussing sensitive topics and to establish a collaborative floor for open discussion. Moreover, women also tend to use hedges when they disclose personal information.

What is notably lacking, however, is to acknowledge the influences of the role of addressees' gender on variations of linguistic differences between groups. There are two studies that show the exceptions in this regard [3, 13]. McMillan et al. explore the gender-related features in terms of four syntactic categories respective in same and mixed groups [3]. They found that female participants use more linguistic devices to connote uncertainty when male speakers are present. Conversely, Brown, observing linguistic behaviors of people in a branch belonging to Mayan culture, found that women tend to use more hedges and weakeners to members of their social groups, namely women in this case. These two typical studies show that how marked the variations are across different contexts and conversational participants.

Taken together, the empirical and theoretical evidence offers a basis and also leaves a gap for further research on features of women's use of hedges, especially with varied contextual factors. Therefore, in the current study two research questions are proposed as following:
1. What are the differences of function-related features of hedges in women's talk in mixed-sex conversations and same-sex conversations?

2. Do the functions of hedges in women's talk in Chinese culture agree with the functions in English?

The first part of this discussion will offer some statistical differences and interpretations of the typical discrepancies in the same- and mixed-sex groups in terms of the use of hedges in four function categories. The second part will include elaborations of some examples to show how and why the subject uses the hedges as interactional facilitative devices in her utterances.

\section{METHODOLOGY}

\subsection{The Podcast Episodes}

The current research data comes from a list of popular Chinese podcast shows, namely, Blow Your Mind, Sparkling Girl, Slightly Open, Fiona's Chat Chat, The Talk Show in Workshop, and Small Talks with Standup Comedians. They are podcasts co-hosted by regular participants in Chinese Mandarin, and guest speaker(s) are invited in some episodes. Moreover, those shows normally discuss a wide range of socially and culturally prevalent topics, such as health, meditation, popular movies and books, and some occupational issues. In terms of discursive features, conversations constructed in podcasts can be positioned between casual style and institutional style [14]. Specifically, podcasts are a type of talk shows characteristic of informality. There might be some planning and preparations about each topic but still, more resemble real-time conversations. However, it should be noted that a defect of using podcasts as data is that there are only audio recordings and thus, facial expressions, gestures or eye contact cannot be analyzed in this paper.

\subsection{The Subjects}

As the research is a case study focusing on one Chinese woman, eight episodes from the mentioned podcasts, all in which the subject was one of the main speakers, are chosen to form the database. The eight episodes are in total 9.5 hours long, roughly 65 minutes each episode on average, 4 of which are mixed-sex conversations (about 4.8 hours) and three same-sex conversations (about 4.7 hours). The subject in this research is a female psychologist and CEO of a startup company in the field of psychological counseling. She is in her thirties and well-educated both in China and abroad. Furthermore, with a male friend, the subject co-hosts the podcast Blow Your Mind, which has been mentioned before and is often invited to other podcast shows as a guest speaker to discuss mental health and gender issues in terms of the psychological sphere. 


\subsection{The Data}

The database contains 150,256 transcribed Chinese characters. There are 424 tokens of hedges used by the subject for the analysis of the present study. The transcript notion in this study is based on Gail Jefferson's transcription conventions (Table 1) [15]. This set of convention is adopted for it is more suitable for analyzing the real-time interactions discussed in this study in detail. In terms of the categorization of functions of hedges, the one given by Coates in her related paper is adopted [8]. However, it should be noted that, as is mentioned previously, this research accepts the conception of hedges and hedging proposed by Fraser and thus, intensifiers are naturally expelled from the discussion [1]. Therefore, linguistic expressions such as actually, really and $I$ ' $m$ sure, included in the first category of Coates's categorization will not be discussed in this study. The four categories of functions of hedges are: the expression of doubt and confidence, sensitivity to others' feelings, searching for the right word and avoiding playing the expert.

Table 1 Transcription Conventions

\begin{tabular}{|c|c|}
\hline[ & overlapping utterance \\
\hline$=$ & $\begin{array}{c}\text { continuous utterance with no noticeable } \\
\text { break or pause }\end{array}$ \\
\hline$()$. & noticeable pause \\
\hline$(0.0)$ & timed pause in tenths of a second \\
\hline Word & underling indicates stress or emphasis \\
\hline Word & upper case indicates especially loud talk \\
\hline$\circ \circ$ & quiet or soft talk \\
\hline Hhh & laughter or breathing \\
\hline$£$ & words that are pronounced in smile \\
\hline$(())$ & transcriber's description of events \\
\hline() & uncertain utterance \\
\hline
\end{tabular}

\section{ANALYSIS}

\subsection{Statistic Results}

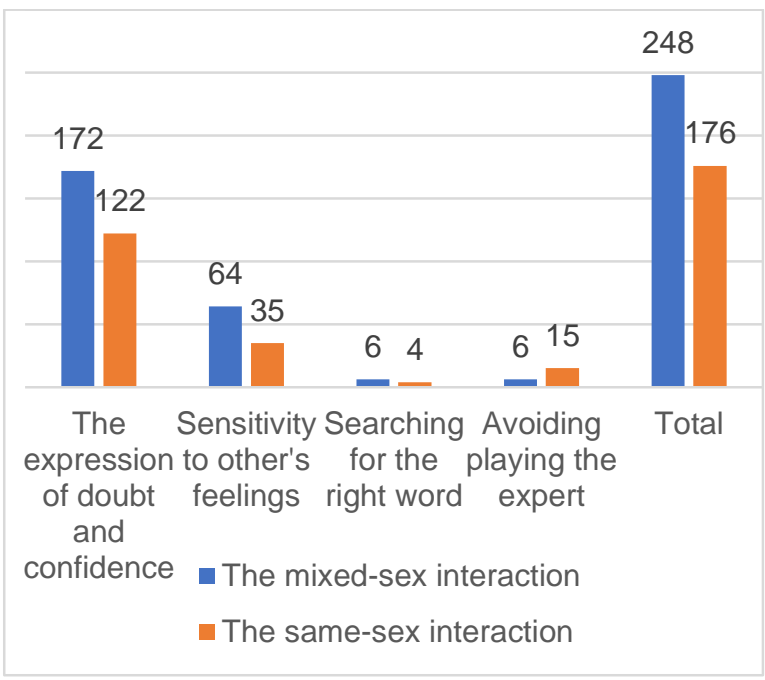

Figure 1 The Numbers of Hedge in the Mixed- and Same-sex Interaction in Functions

Judging from Figure 1, overall, the subject shows a marked difference in terms of using hedges in mixed-sex interactions and same-sex interactions in the context of Chinese podcast shows. The subject shows more hedging behaviors in the conversations where men are present. This result disagrees with what Brown finds in a different cultural and social context in which women tend to use more hedges and weakeners to people of their sex [13]. To interpret this, the Chinese cultural norms would be one of the explanations. However, this aspect involves too many subfactors to be considered and thus, it would be too rash to draw some descriptive conclusions in this case study. Another hypothesis that could be used to explain the result would be Lakoff's claim that hedges and hedging are characteristic of highly informal and casual interactions [2]. While Brown's data is collected from very natural, casual conversations, the present study's data is extracted from podcast shows, a semi-formal interaction style [14]. There is a possibility that the formality of the interaction style produces the discrepancy.

Regarding the functions of hedges, all the four functions proposed by Coates display in the database, which runs counter to Lakoff's statement that women use linguistic devices as hedges to express only tentativeness and unassertiveness. Indeed, hedges can be featured as highly multi-functional in the context of both mixed- and same-sex conversations, also in line with Holmes' study [10].

In terms of the first category, the subject employs considerably more hedging linguistic devices to express her uncertainty and confidence in the mixed-sex podcast shows. This may be seen as partly similar to one of 
Dixon and Foster's conclusions that speakers use epistemic sort of more when they have conversations with male partners [16]. An intuitive hypothesis that is likely to be accepted would be that "men receive a higher proportion of 'face-sensitive' communication than women." Nevertheless, in light of the data per se, a more reasonable explanation for this needs to take the topics and contents of the podcast episodes into consideration. Among the four woman-to-woman episodes, two of them are in an interview-style focusing on the subject's personal life and career experience, which naturally involve less necessity to employ epistemic hedges. By contrast, all the four mixed-sex conversations, discussing sensitive topics like mental health and feminism, require speakers to carefully attenuate or mitigate their commitment to the truth of the propositions [8]. This can also be the reason to elucidate what happens in terms of the second category: sensitivity to others' feelings.

It is also notable that the subject uses more hedges to avoid being an expert in same-sex interactions. This is the sole category of which the number of hedges employed by the subject in woman-to-woman talks surpasses that in the mixed-sex talks. It demonstrates much agreement with Coates' claim that female speakers tend to construct a collaborative floor to draw the partners into the conversation and construct the talk together [8]. The avoidance of playing the expert is a crucial way to reduce social distance and arrive at a collaborative floor open to every conversational participant.

\subsection{Analysis of Multiple Functions of Hedges}

As is emphasized by many linguists, the attempt to explore relationships among hedges, gender features and communication cannot be done if functions and contextual variables are not taken into account $[3,8,10]$. Thus, on the basis of the results in the previous part, this part will illustrate how hedges are used to execute various functions showing in Figure 1 by analyzing examples in concrete social and textual contexts, in which a brief indication of the context of each excerpt is given to facilitate interpretation and hedges are in bold.

\subsubsection{The Expression of Doubt and Confidence}

To use hedges to express the speakers' lack of commitment to the truth of the proposition is the basic function of hedging behaviors [1]. This is indeed true for the data of the present study, as this category consists of a large proportion of hedges used by the subject. In example (1), the subject (S) and her male co-host (M) are talking about the relationship between women's beauty and social norms and values. $\mathrm{S}$ mentions that Korean women have plastic surgery to meet socially stereotypical standards for women. However, her utterance is greeted with doubt by $\mathrm{M}$.

\section{Transcript 1}

M: ni zen me pan duan de? ni zen me jiu pan duan chu han guo zhe xie ta bu shi zi ji xiang zhui qiu zhe ge, sui ran wo wo zhi jue gan jue ye shi.

How do you know the reason why Korean women have plastic surgeries is not because they, themselves, want to do that? Although, I think you are right intuitively.

S: emmm, dang ran shi you zhe ge ke neng xing le, dan wo jue de (.) wo ye zai xiang zhe na li lai de, ke neng shi ji yu he yi xie han guo peng you liao tian.

Emmm, of course, there is a possibility that they have the surgeries because they want to. But I think...I don't know where my idea comes from. Probably based on my discussion with some Korean friends.

\section{M: Yeah.=}

Yeah.

$\mathrm{S}:=$ huo zhe zhe me jiang, ni kan han guo de zong yi, sui ran wo kan de ye bu shi hen. duo.

Or in other words, you can get some idea from Korean entertainment shows. Though, I didn't watch them a lot.

Females' beauty, plastic surgeries and social norms for women are sensitive and controversial topics in the Chinese culture. When people discuss this kind of issue, they are highly likely to mitigate the force of the utterance, or it will be very difficult to express their ideas, especially in the context of podcast shows where many 'invisible' addressees exist [8]. After her partner expresses his doubt, $\mathrm{S}$ begins her utterance by emmm indicating the tentativeness of her earlier proposition. Following that, she partly agrees with the man by using possibility as a hedge. However, she continuously attempts to explain her own views but signals her uncertainty with "I think" and "probably." Moreover, it is worth noting that at the end of this example, $\mathrm{S}$ effectively attenuates the force of her utterance by a whole sentence "Though, I didn't watch them a lot." The sentence is directed by another kind of hedging device: concessive conjunction.

In addition to prevalent forms of hedges, such as wo jue de (I think) and ke neng (probably) to signal uncertainty in Chinese, words like yi ban (generally) and da gai (roughly) are prominent linguistic devices to indicate the speakers' unassertiveness. In the database of the study, it should be noted that the subject is highly likely to use this kind of hedge in a particular context, in which she is asked to talk about her job and introduce basic knowledge of psychological counseling. There are two typical examples extracted from the utterance of the subject. Noticeably, in example (3), the speaker 
expresses her doubt and uncertainty by first premodifying that psychological counseling progress is complicated, following an apparent pause and signaling her uncertainty by $y i$ ban (generally). All the three factors (the premodification, the pause and the hedge word) contribute to communicating S's uncommitting to her proposition.

\section{Transcript 2}

[trying to answer interviewer's question about how $\mathrm{S}$ define herself in public]

S: ru guo zhu chi ren zai hu dong shang jie shao de shi hou, ru guo ta ba wo ding yi. weixin li zi xun shi, wo $\boldsymbol{Y I} \boldsymbol{B} \boldsymbol{A N}$ dou hui rang ta xiu gai cheng wei jian dan xin li de chuang shi ren.

If some interviewers in public activities introduce me as a psychologist, generally I ask him to change the introduction and define me as the co-founder of Jian Dan Xin Li ((the company's name started up by the subject)).

\section{Transcript 3}

[introducing how psychological counseling works]

S: zhe shi ge fu za de wen ti. (.) jiu shi yi ban xin li zi xun shi you gu ding de shi jian, gu gu ding de di dian, yi ji gu ding de pin lv lai jin xing de.

It is complicated. Well psychological counseling is done by a regular timetable and frequency and in a regular place.

\subsubsection{Sensitivity to Others' Feelings}

Damping down the force of one's speech act is proposed as one of the major pragmatic competencies by Fraser, to show sensitivity to others' feelings and be viewed as polite or not offensive [1]. It is closely related to the theory of politeness [17]. The theory argues that individuals have face needs and tend to protect these needs in interactions. Example (4) is taken from a mixed-sex conversation to discuss how humor improves mental health. The excerpt is taken from a point where the interviewer asks the subject of this study to introduce the psychological counseling service of her company. After a brief introduction, S ends her utterance by saying:

\section{Transcript 4}

S: £ ru guo wan yi da jia you xu yao de hua £, huan ying da jia shi yong wo men de fu wu.

If, in case, you ((the listeners)) need to have some counseling, welcome to use our service.

The speaker uses compound hedges ru guo (if) and wan yi (in case) together to disarm her imposition on the addressees. Reasonably, she knows that psychological counseling is still a very new, sensitive and even unwelcome field for the public in China. Thus, she attenuates her utterance instead of bluntly impose on the addressees. By doing this, $\mathrm{S}$ protects the negative face of the addressees.

In the next example, after one of the speakers (A) participating in that episode narrates her personal experience in a very horrible relationship, she asks the subject a sensitive question but in a smiling voice. Nevertheless, the subject feels the apparent need to mitigate her responding utterance as she knows it might include highly charged content.

\section{Transcript 5}

A: suo yi, wo zhe shi £you si de ge er mo zong he zheng ma£, shi you ma?

So, do I have the Stockholm syndrome, do I?

S: wo jue de zhi shao ni ke yi jian li yi ge biao zhun Shou xian wo zi ji jue de, si hu, you ke neng, ta ke neng ji bus hi yi ge hao ren, ye bu shi huai ren, dan wo jue de zhi shao, ta bu shi yi ge (.) qing xu zhi li shang hen jian kang de ren...

I think, at least, you can set up a standard ((to evaluate a person)). First, personally, I think, it seems there is possibility...probably he is not a good person and not a bay person either. But I think he is not very mentally health.

S's utterance has many hedging behaviors to mitigate her evaluative comments on the person in A's story. The story is a self-disclosure speech act that puts $\mathrm{A}$ in a vulnerable position and her attitudes toward the person she talks about are not very clear. Given that, S's mitigative attempts are a way to protect A's face by giving A space to join in her evaluation. Meanwhile, she keeps the chance to wriggle out of accusation for negatively evaluating the person in A's story. In particular, in her second sentence, $\mathrm{S}$ uses four hedges one after another at the beginning, which typically shows her consideration to care about the addressee's feelings.

\subsubsection{Searching for the Right Word}

Hedges also allow speakers to have time to struggle and come up with appropriate and accurate words to express their ideas. This function of hedge is particularly employed by female speakers [8]. In contrast to Coates's examples like "sort of" and "you know" which are typically used as hedges to signal the act of searching for the right word in English, in the current database, there are specific Chinese expressions used by the subject to play the role of this function. The most prominent expression is zen me jiang (how to say). In the following example, the conversational parts are discussing how to deal with sufferance in the occupational sphere, and one female speaker asks the 
subject how to understand the relationship between this kind of sufferance and the ability to be resilient.

\section{Transcript 6}

S: wo zi ji (.) jue de, wo zen me jiang,(2.0) wo jue de zhe shi liang ge fang xiang.

Personally, I think...how to say, I think it can be viewed as two different aspects.

This example is in line with Coates' idea that there is usually a pause after the hedge if the hedge indicates that the speaker is having trouble with words but not giving up his/her turn. It is worth noticing that the speaker achieves her interactional goal by using zen me jiang (how to say), for even though she pauses for 2 seconds, which is a long pause in any conversation, the other participants still wait for her instead of taking the turn. Note that in example (5), the subject's use of four hedges one after another is also a way to signal her struggle with appropriate words.

\subsubsection{Avoiding Playing the Expert}

In the process of having conversations with other participants, women usually tend to create a collaborative floor and draw others into the conversations [8][18]. One way of achieving this aim is not to take on the role of expert by using hedges of mitigative quality. If conversational parts can express their parts tentatively, they might be able to minimize the social distance between each other and construct the talk together. Particularly, the author wants to argue that the avoidance of playing the expert is of greater importance if there is a literal expert present in the interaction. Example (7) is an excerpt constructed by a person interested in psychology (B) and an expert in this field, namely the subject of this study. They are talking about the way people seek psychological help.

\section{Transcript 7}

S: wo yi ban hui jian yi zhao xin li zi xun shi huo zhe zhao jing shen ke yi sheng, na ge. dui ni rong yi jiu zhao na ge.

Generally, I suggest people to go to a psychologist or a psychiatrist, whoever he can. get accessed to easily.=

B: =wo jie chu dao de da bu fen wo men de yong hu ke neng shi ba qing gan bo zhu. dang zuo xin li yi sheng, wen ta wo gai zen me ban.

Most of the users of my service might view those KOLs in the field of relationships on the internet as psychologists and ask for their opinions.

$\mathrm{S}$ : dui, ye mei shen me bu hao de, wo jue de zhe ge yao yuan yuan hao guo ni bu qu qiu zhu.

Yeah, that's ok. I think it's still better than not asking for help at all.
B: emmm, dui, na ke neng shi ta xing dong de yi ge fang shi.

Emmm, yeah, probably, that is the way he chooses to ask for help.

$\mathrm{S}: d u i$.

yeah.

S's first utterance is hedged by yi ban (generally) to show her attitude that she does not hold this position firmly and wants to discuss. This attempt succeeds, indicated by B's quickly taking the turn to express her disapproval that some people seek help from non-professionals (her disapproval can be signaled by the emmm in her second turn, which means she hesitates and choose to change her position from disapproval to approval). Even though B expresses a negative opinion, she still chooses to use da bu fen (most) and ke neng (might) to hedge her opinion, inviting $\mathrm{S}$ to continue to express her views.

In turn, $\mathrm{S}$ acknowledges the way $\mathrm{B}$ mentions with a typical hedge wo jue de (I think) and notably, after a hesitative "emmm", B agrees with S explicitly and responds to S's acknowledgment by offering her new hypothesis. Finally, they arrive at a consensus. The process of how they arrive at a consensus is also the process of how women construct a collaborative floor and keep the conversations open to each other to express different opinions [8].

\section{CONCLUSION}

Research on features of linguistic devices used in women's speech concerning gender and communication is no less than complex and challenging. This case study has shown both quantitative results in terms of functions of hedges in women's speech and an elaborative analysis of how women use hedges to execute their interactional functions in conversations. Based upon the two-part discussion, it should be reasonable to conclude that while women today, as Lakoff observed almost 50 years ago, do use hedges to signal their tentativeness, they also use them to show their negative and positive politeness. In other words, they are more likely to create interpersonal closeness in interactions by taking care of others' feelings and drawing others into the conversations to express different opinions. In line with what Coates proposed, this study views women's ability to deploy these functions of hedges successfully as a kind of advantage instead of a weakness of women [8].

However, the quantitative results and discourse analysis are provisional. Thus, some limitations and implications for future studies should be mentioned. First, the present study is a case study focusing on the speech of one female Mandarin speaker, which means the sample size is small and a larger number of subjects and data of speech should be involved to produce a 
theoretical generalization. Secondly, the data was collected from podcast shows which is, to some extent, a formal context for conversations. Therefore, some specific concerns like the "invisible" audience have been taken into account through the analysis. Those factors may not be necessary for discussions on everyday talk and thus, explorations in more varied contexts could be done in the future. Last but not least, future research in this area of gender and communication in Chinese could focus more closely on examining the functions of specific hedging linguistic devices in terms of their gender-related features.

Overall, the current research is remarkably consistent with the previous study on women's language usage, especially the functions of hedges. In terms of the contribution, this research might help establish the framework for interpreting women's linguistic activities and recreate women's social role in Chinese culture by updating their communicative features to the public.

\section{REFERENCES}

[1] Fraser, B. (2010). Pragmatic competence: The case of hedging. New approaches to hedging, 1534.

[2] Lakoff, R. (1973). Language and woman's place. Language in society, 2(1), 45-79.

[3] McMillan, J. R., Clifton, A. K., McGrath, D., \& Gale, W. S. (1977). Women's language: Uncertainty or interpersonal sensitivity and emotionality?. Sex roles, 3(6), 545-559.

[4] Bernard, J. The sex game. New York: Atheneum, 1972.

[5] Lauwereyns, S. (2002). Hedges in Japanese conversation: The influence of age, sex, and formality. Language Variation and Change, 14(2), 239-259.

[6] Priesler, B. (1986). Linguistic sex roles in conversation: Social variation in the expression of tentativeness in English.Berlin: Moutin de Gruyter.

[7] Carli, L. L. (1990). Gender, language and influence. Journal of Personality and Social Psychology. 59, 941-951.
[8] Coates, J. (2013). 'So I Mean I Probably...': Hedges and Hedging in Women's Talk [2003]. In Women, Men and Everyday Talk (pp. 31-49). Palgrave Macmillan, London.

[9] Coates, J. (1997). Women's friendships, women's talk. Gender and discourse, 245262.

[10] Holmes, J. (1990). Hedges and boosters in women's and men's speech. Language \& Communication, 10(3), 185-205.

[11] Precht, K. (2008). Sex similarities and differences in stance in informal American conversation 1. Journal of Sociolinguistics, 12(1), 89-111.

[12] Dubar, T. (2012). Gender-Related Features in the Use of the Hedge" You Know"-A Case Study of Conversations on the Radio Station, London's Biggest Conversation 97.3 FM.

[13] Brown, P. (1980). How and why are women more polite: Some evidence from a Mayan community. In Women and language in literature and society (pp. 111-136). Praeger.

[14] Ilie, C. (2001). Semi-institutional discourse: The case of talk shows. Journal of pragmatics, 33(2), 209-254.

[15] Jefferson, G. (2004). Glossary of transcript symbols with an introduction. Pragmatics and beyond new series, 125, 13-34.

[16] Dixon, J. A., \& Foster, D. H. (1997). Gender and hedging: From sex differences to situated practice. Journal of psycholinguistic research, 26(1), 89-107.

[17] Brown, P., \& Levinson, S. C. (1978). Universals in language usage: Politeness phenomena. In Questions and politeness: Strategies in social interaction (pp. 56-311). Cambridge University Press.

[18] Edelsky, C. (1981). Who's got the floor?. Language in society, 10(3), 383-421. 